\begin{abstract}
Annual Report
Direct Measurement of U235 and Pu239 in Spent Fuel Rods with Gamma-Ray Mirrors

OR10-GammaMirror-PD2Lb

LL10-GammaMirror-PD2Lb
\end{abstract}

Lab:

Project PI:

Primary Author:

Contributors:

Date:

ORNL, LLNL

Klaus Ziock (ORNL), Mike Pivovaroff (LLNL)

Klaus Ziock

J.B. Alameda, N.F. Brejnholt, T.A. Decker, M.-A. Descalle, M. Fernandez-Perea, R.M. Hill, R.A. Kisner, A.M. Melin, B.W. Patton, J. Ruz, R. Soufli

9/30/13

Approved for public release.

Distribution is unlimited. 


\title{
Direct Measurement of U235 and Pu239 in Spent Fuel Rods with Gamma-Ray Mirrors
}

\author{
OR10-GammaMirror-PD2Lb
}

\section{INTRODUCTION}

The amounts of fissile $\mathrm{Pu}$ and $\mathrm{U}$ in spent nuclear fuel are of primary concern to the safeguards community. In particular, there are issues when safeguards transitions from an item accountancy basis (such as fuel bundles) to a fissile material mass basis as occurs when spent fuel enters a reprocessing plant. Discrepancies occur because item accountancy requires estimating the content of fissile material using indirect techniques such as the fuel burn-up and item-level measurements of radiation emissions from fission by-products. Direct measurement of the fissile content by monitoring line emissions from fissile species themselves is impossible because the lines are much weaker than those emitted by shorter-lived isotopes in the fuel. The goal of this project is to develop a technique to directly measure these weaker lines despite the presence of overwhelming radiation from other isotopes. This is achieved by using gamma-ray mirrors as a narrow band-pass filter. The mirrors reflect only energies of interest toward a HPGe detector that is shielded from direct view of the spent fuel and its fierce emissions. This can significantly improve the reliability with which the mass of fissile material is tracked.

\section{TECHNIQUE}

Reflective $\mathrm{x}$-ray telescopes and optics based on grazing incidence techniques have been used in astrophysical research for several decades. Above $\sim 40 \mathrm{keV}$, multilayer coatings are required to achieve high throughput in practical configurations. Satellite missions have recently shown this approach works up to $~ 80 \mathrm{keV}$ [1]. Multilayers can be thought of as a synthetic Bragg crystal composed of hundreds of alternating layers of two or more materials. The period, $d$, of the multilayer satisfies Bragg's law, $m \lambda=2 d \sin \theta$, where lambda is the photon wavelength, $m$ is the order (usually $m=1$ ) and $\theta$ is the incident angle of the photon. In earlier years of this research program, we have shown that multilayers can work up to energies of $386 \mathrm{keV}$ [2,3]. Recent, independent, LDRD work at LLNL has demonstrated that multilayers can work well up to $650 \mathrm{keV}$ [4]. The key for achieving good reflectivity is selecting an appropriate multilayer system and depositing coatings with periods as small as $1.0-1.5 \mathrm{~nm}$.

The goal of this project was to validate the concept that an optic based on multilayer coatings could be used to reflect only a narrow range of energies around a specific emission line from the spent fuel onto a detector; for instance the $186-\mathrm{keV}$ line from ${ }^{235} \mathrm{U}$ or the $375-\mathrm{keV}$ line from

${ }^{239} \mathrm{Pu}$. By passing only a narrow range of energies around one of these lines, the problem of overwhelming the detector with high count rates from out-of-band photons would be eliminated.

\section{EXPERIMENTAL PROGRAM}

To validate this concept, we have used a stepped approach. In previous years we demonstrated that a single emission line ( $59 \mathrm{keV}$ from ${ }^{241} \mathrm{Am}$ ) from a multisource region could be reflected onto a detector shielded from direct view of the sources. We then designed and fabricated an optic and collimator system to test the concept with spent nuclear fuel pins at the Irradiated Fuels Examination Laboratory (IFEL) at ORNL. Installation of the system and data on different fuel pins were collected this year. 
Fig. 1. shows a rendered image of the experiment fielded at the IFEL including the mirror assembly, its manipulator, and a collimator to limit flux reaching the shirtsleeve environment in which the measurements were conducted. A stack of
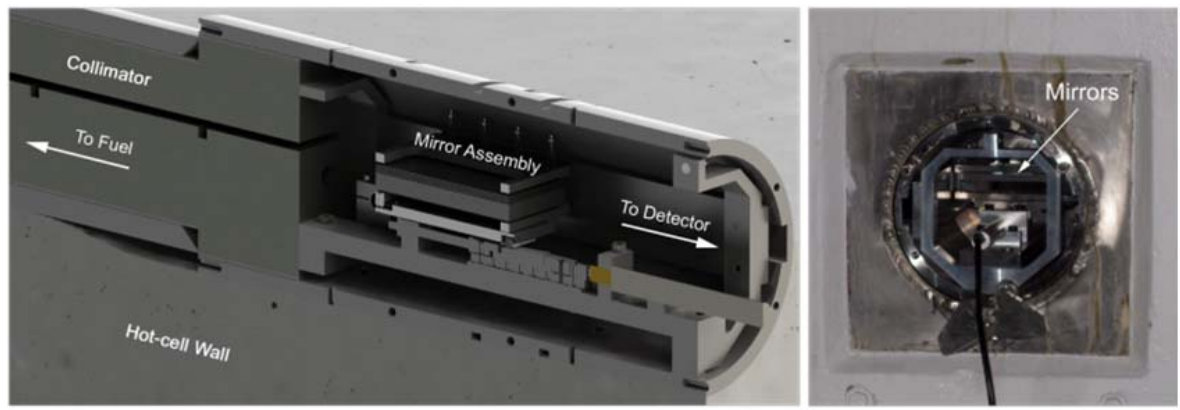

Fig. 1. Schematic of hot cell experiment (left). Photograph of the system installed in a port of the hot-cell (right).

five flat mirrors with coatings that have a nominal bi-layer spacing of $1.5 \mathrm{~nm}$ was used to deflect the radiation of interest onto a one-cm-thick, position-sensitive HPGe detector. In these first-ever measurements, we targeted the atomic K-shell fluorescence lines from U both because they are excited by the other radiation, providing a stronger first signal, and because the mirrors will deflect lower energy lines through larger angles making separation from the primary beam greater. At the IFEL we were limited to deflection lengths of $\sim 3 \mathrm{~m}$, favoring lower energy lines for maximum separation between the deflected and primary beams.

The results from the experiment clearly show that the mirrors perform as expected and that the technique is viable. The counts distributed across the face of the detector are shown in Fig. 2. In 2A the direct beam

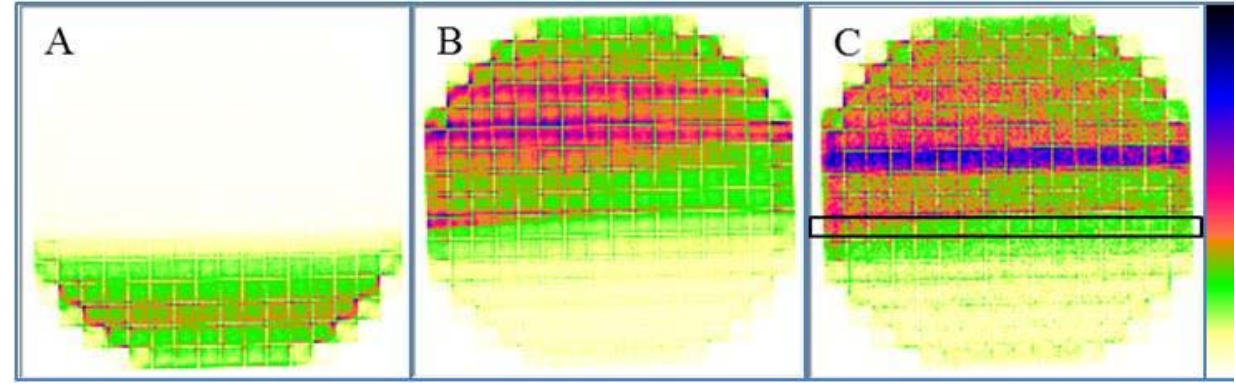

Fig. 2. Full face detector response to the spent fuel (A) without blocking the primary beam, (B) with the beam blocked and the mirror set to an angle of $0.15^{\circ}$, (C) same as (B) but with an energy window set (92-112 keV) to see the $\mathrm{K}$ emission lines from $\mathrm{U}$. from the port overwhelms the on-line analysis code with the beam striking the lower portion of the detector giving a count rate of $\sim 21 \mathrm{kHz}$. When this beam is blocked, and the mirror is positioned to its lowest angle $\left(0.15^{\circ}\right)$, the rate on the unblocked region of the detector falls to about twice the room background when no rod is in front of the port, while the overall detector rate is just $570 \mathrm{~Hz}$. When the mirror angle is changed to deflect the K-shell lines above the lead block (Fig. $2 \mathrm{~B} \& \mathrm{C}$ ) a line structure is seen on the face of the detector and the spectrum from that region (Fig. 3) clearly shows the fluorescence lines from the rod. A more detailed analysis to determine the relative intensities of the different lines is given in Table 1 and shows excellent agreement with the expected line strengths.

Table 1: Line Intensities

\begin{tabular}{cccc}
\hline U Line & $\begin{array}{c}\text { Expected } \\
\text { [\%] }\end{array}$ & $\begin{array}{c}\text { Measured } \\
\text { [\%] }\end{array}$ & $\begin{array}{c}\text { Deviation } \\
\text { [\%] }\end{array}$ \\
\hline \hline $\mathrm{k} \alpha_{1}$ & 100 & 100 & 0 \\
$\mathrm{k} \alpha_{2}$ & 61.9 & 62.2 & 0.3 \\
$\mathrm{k} \beta_{1}+\mathrm{k} \beta_{3}$ & 33.6 & 34.4 & 0.8 \\
$\mathrm{~K} \beta_{2}$ & 12.3 & 11.3 & 1.0
\end{tabular}

\section{RESULTS, DISCUSSION AND CONCLUSIONS}

The results obtained at the IFEL are extremely encouraging. They unequivocally show that the approach works in the demanding environment posed by spent nuclear fuel. Fears that we would 
be unable to separate the deflected beam from the primary beam or that scattered radiation from the mirror would totally dominate the spectra were unfounded.

The optic used was the simplest possible approach, comprising just a 5-mirror stack of planar mirrors. However, it did not provide any focusing, nor was the multilayer spacing specifically tuned to maximize the response for a single emission line. A more complex optic, for example one using curved substrates that would allow focusing or improved "nesting” for higher

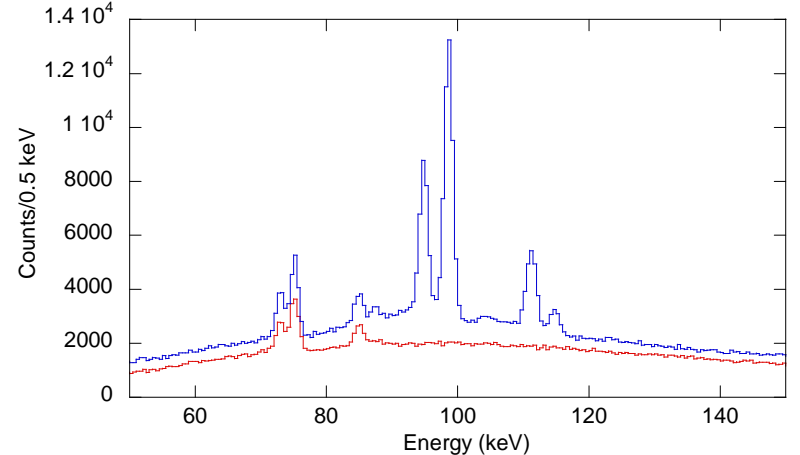

Fig. 3. Spectra from the blue region of the detector in Fig. 2C (blue) and a similarly-sized area (black rectangle in 2C) just above the beam stop (red). throughput, should be able to significantly increase the signal reaching the detector. We must also work to optimize shielding to reduce high energy photons from reaching the detector. Both efforts should improve the signal-to-noise ratio obtained with the system. Further, one can take advantage of the inherent Compton imaging capabilities of the detector used in the experiment to remove some of the background caused by higher energy gamma-rays reaching the detector.

\section{PATH FORWARD}

The first task will be to perform another set of measurements to better understand both the noise and signal components seen previously. While the measurements presented above took very little time to see the K-shell lines, the SNR obtained with the system was not as good as that obtained by a prior experiment using a very tightly collimated beam and a long exposure to look at the fuel directly [5]. Through the use of a single mirror and tighter collimation we will be able to better characterize both the signal and the noise reaching the detector. Once a better understanding is achieved, future efforts will focus on optimizing the design of the gamma-ray optic and an effective collimation and shielding configuration tailored around the geometry of HPGe detector.

\section{PRESENTATIONS AND PUBLICATIONS}

1. M. Fernández-Perea et al. "Ultra-short-period WC/SiC multilayer coatings for X-ray applications," Nuclear Instruments and Methods A, 710, 114 (2013).

2. M. Fernández-Perea et al. "Physics of Reflective Optics for the Soft Gamma-Ray Photon Energy Range," Physical Review Letters, 111, 027404, (2013).

3. M. J. Pivovaroff et al. “Gamma-ray Mirrors for Direct Measurement of Spent Nuclear Fuel,” prepared for the 2013 INMM Conference*, [LLNL-PROC-639345] (July 2013).

* paper not presented due to restrictions on travel; manuscript being prepared for submission to Nuclear Instruments and Methods A

\section{REFERENCES}

1. F. A. Harrison et al. "The Nuclear Spectroscopic Telescope Array (NuSTAR) High-energy X-ray Mission,” Astrophysical Journal, 770:130 (2013).

2. M. Fernández-Perea et al. "Physics of Reflective Optics for the Soft Gamma-Ray Photon Energy Range," Physical Review Letters, 111, 027404, (2013).

3. M. Fernández-Perea et al. "Ultra-short-period WC/SiC multilayer coatings for x-ray applications,” Nuclear Instruments and Methods A, 710, 114 (2013).

4. M. J. Pivovaroff et al. "High reflectivity multilayers for hard X-ray and soft gamma-ray applications," SPIE Optics + Photonics 2013 [LLNL-PRES-643015] (August 2013).

5. W. S. Charlton et al. "The Use of Self-Induced XRF to Quantify the Pu Content in PWR Spent Nuclear Fuel,” Proceedings of the 31st Annual Meeting of ESARDA (2009). 